\title{
Palladium-Catalyzed Asymmetric Allylic Alkylation Reaction of 2-Monosubstituted Indolin-3-ones
}

\author{
Tie-Gen Chen ${ }^{a}$ \\ Ping Fanga \\ Xue-Long Hou ${ }^{* a, b}$ \\ Li-Xin Dai ${ }^{a}$ \\ a State Key Laboratory of Organometallic Chemistry, Shanghai \\ Institute of Organic Chemistry, Chinese Academy of Sciences, \\ 345 Lingling Road, Shanghai 200032, P. R. of China \\ b Shanghai-Hong Kong Joint Laboratory in Chemical Synthesis, \\ Shanghai Institute of Organic Chemistry, Chinese Academy of \\ Sciences, 345 Lingling Road, Shanghai 200032, P. R. of China \\ xlhou@sioc.ac.cn
}

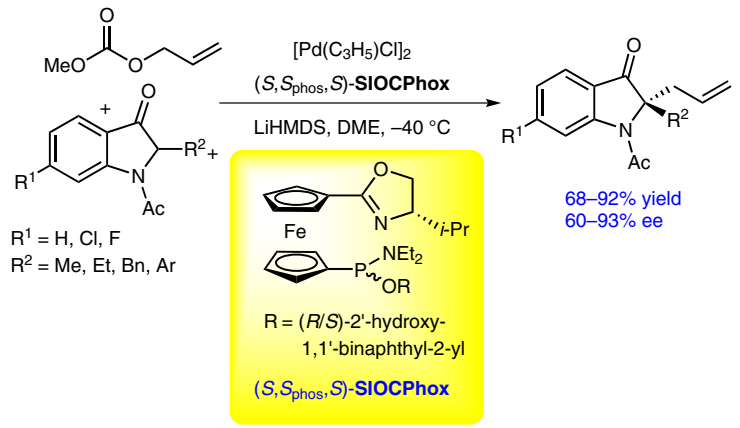

date several methodologies have been developed, ${ }^{7}$ however, the only successful protocol that proceeds through asymmetric catalysis involves addition reactions to the $\mathrm{C}-\mathrm{N}$ double bond of 2-substituted $3 \mathrm{H}$-indol-3-ones ${ }^{7 \mathrm{~m}-\mathrm{r}}$ or their analogues. ${ }^{7 \mathrm{~s}}$ Only one report has appeared for the $\alpha$-alkylation of 2-substituted indolin-3-ones catalyzed by a chiral catalyst, and the reaction proceeds with moderate enantioselectivity. ${ }^{7 t}$ Palladium-catalyzed asymmetric allylic alkylation (AAA) is one of the most important reactions for the enantioselective construction of $\mathrm{C}-\mathrm{C}$ and $\mathrm{C}$-hetero atom bonds in organic synthesis. ${ }^{8}$ However, a few examples of $\alpha$ amino ketones being used in the reaction as prenucleophiles via the enolates derived from ketones and carboxylic acid have been successfully demonstrated. ${ }^{7 t, 9,10 \mathrm{~d}-\mathrm{f}, \mathrm{i}}$ In the course of our research on palladium-catalyzed AAA during recent years, ${ }^{10}$ we have successfully used different types of enolates as nucleophiles. ${ }^{10 \mathrm{~d}-\mathrm{f}, \mathrm{i}}$ In this paper, we would like to \section{brevianamide $A,{ }^{2}(+)$-aristotetelone, ${ }^{3}$ isatisine $A,{ }^{4}$ hinckden-
tine $A,{ }^{5}$ and others ${ }^{6}$ (Figure 1 ). Therefore, their synthesis has attracted much attention from synthetic chemists. To \\ The 2,2-disubstituted indolin-3-one moiety is an im- portant structural motif in many natural products and compounds of pharmaceutical interest, such as austamide, ${ }^{1}$ brevianamide $A,{ }^{2}(+)$-aristotetelone, ${ }^{3}$ isatisine $A,{ }^{4}$ hinckden-
tine $A,{ }^{5}$ and others ${ }^{6}$ (Figure 1 ). Therefore, their synthesis has attracted much attention from syn \\ Accepted after revision: 08.08.2014 \\ Published online: 29.09 .2014 \\ Abstract An efficient and practical method was developed for the syn- metric allylic alkylation. Enantioselective construction of quaternary \\ Key words palladium, allylic substitution, asymmetric catalysis, indo- lines, quaternary carbon}

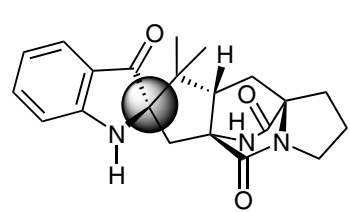

brevianamide $\mathrm{A}$

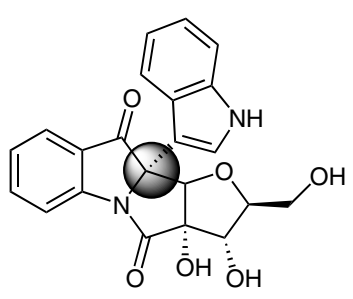

isatisine $\mathrm{A}$

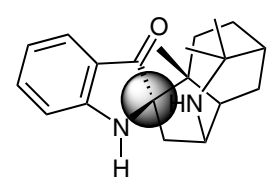

(+)-aristotetelone

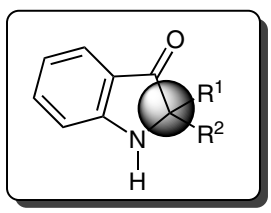

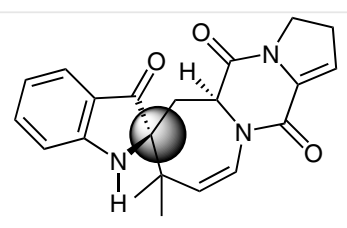

austamide

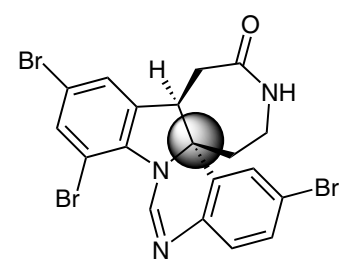

hinckdentine A

Figure 1 Some natural products containing the indolin-3-one substructure with an $\alpha$-quaternary carbon center 
report our further studies on the use of 2-substituted indolin-3-ones as prenucleophiles to construct chiral quaternary centers by palladium-catalyzed AAA. ${ }^{10 \mathrm{~b}, 11}$

Initially, the reaction of 1-acetyl-2-benzylindolin-3-one (1a) and allyl methyl carbonate (2a) was carried out in the presence of catalytic amounts of $\left[\mathrm{Pd}\left(\mathrm{C}_{3} \mathrm{H}_{5}\right) \mathrm{Cl}_{2}\right.$ and $(R)$ BINAP, using LiHMDS as base in tetrahydrofuran (THF) at $-40^{\circ} \mathrm{C}$. The reaction afforded the product 2,2-disubstituted indolin-3-one 3a in $83 \%$ yield and 38\% ee (Table 1, entry 1 ). Encouraged by this result, the reaction parameters were investigated (Table 1 ).

It was found that good yield was achieved, although enantioselectivity was not high, with ligands L2-L5 (Figure 2 and Table 1 , entry $2-5$ ). The ee value decreased significantly when allyl tert-butyl carbonate (2b) was used (Table 1, entry 6 vs 2). A screen of SIOCPhox ligands developed by our group ${ }^{10}$ revealed that the ligand $\left(S, S_{\text {phos }}, S\right)$-SIOCPhox $\mathbf{L 6}$ afforded better results with respect to both yield and enantioselectivity (entry 7) whereas the other SIOCPhox ligands L7-L14 provided inferior results (entries 8-15).

On the basis of the above results, the effects of solvents and bases using $\left[\mathrm{Pd}\left(\mathrm{C}_{3} \mathrm{H}_{5}\right) \mathrm{Cl}\right]_{2}$ and $\left(S, S_{\text {phos }}, S\right)$-L6 as catalyst was investigated. It can be seen that dimethoxyethane (DME) gave better results (Table 1, entry 17) among various solvents screened, including toluene, DME, $\mathrm{Et}_{2} \mathrm{O}$ and $\mathrm{CH}_{2} \mathrm{Cl}_{2}$ (entries 16-19), whereas LiHMDS was found to be the most suitable base (entry 17). Both yield and ee value decreased significantly when NaHMDS, KHMDS, or LDA was used as base (entries 20-22), and $\mathrm{NaH}$ and $t$-BuONa gave only trace amounts of product (not shown). The use of some common additives, such as $\mathrm{LiCl}, \mathrm{ZnCl}_{2}$, and $\mathrm{Bn}(\mathrm{Et})_{3} \mathrm{NCl}$, did not improve the yield and/or ee (entries 23-25).
Under the optimized conditions, the substrate scope was then explored; the results are summarized in Table 2. Generally the reactions proceeded smoothly for all substrates, with yields of $62-99 \%$ and ee values of $68-92 \%$ being obtained for substrates with benzyl (entry 1 ), methyl (entry 2), ethyl (entry 9), phenyl (entry 7), o-Br substituted phenyl (entry 8) and benzyl with electron-withdrawing or electron-donating substituent on phenyl group (entries 1013 ) at the $\alpha$-position to the carbonyl. The reaction was also suitable for indolinones with $\mathrm{Cl}$ or $\mathrm{F}$ at the 6-position (entries 3-6). It should be noted that the reactions of $\mathbf{1 g}, \mathbf{1 h}, \mathbf{1 1}$, and $\mathbf{1 m}$ afforded the corresponding products in lower yields (not shown), however, the yields were improved when the reaction was carried out at 0 or $15^{\circ} \mathrm{C}$. Substrate $1 \mathrm{~g}$ afforded the product $\mathbf{3 g}$ in $86 \%$ yield with $92 \%$ ee and substrate $\mathbf{1 h}$ gave $\mathbf{3 h}$ in $92 \%$ yield and $87 \%$ ee at $15{ }^{\circ} \mathrm{C}$ (entries 7 and 8), whereas $\mathbf{1 1}$ gave $\mathbf{3 1}$ in $93 \%$ yield and $84 \%$ ee, and $\mathbf{1 m}$ provided $3 \mathrm{~m}$ in $83 \%$ yield and $68 \%$ ee at $0{ }^{\circ} \mathrm{C}$ (entries 12 and 13 ). Substrate 1n, with a furan-2-ylmethyl substituent, provided $3 n$ in $80 \%$ yield and $76 \%$ ee (entry 14 ). It should be pointed out that the product $\mathbf{3 h}$ could be used as a key intermediate in the total synthesis of hinckdentine A. ${ }^{5 b, c}$

The absolute configuration of $\mathbf{3 a}$ was determined as $R$ by comparing the sign of the optical rotation of the product with that previously reported.7t

In summary, we have realized the palladium-catalyzed asymmetric allylic alkylation of 2-substituted indolin-3ones with allyl methyl carbonate in good yields and with good enantioselectivities. A chiral quaternary carbon center was also constructed during the reaction. These synthetically useful products could find applications in organic synthesis. ${ }^{5 b, c}$ Investigations to extend the scope of the reaction and develop further its application in organic synthesis are in progress.

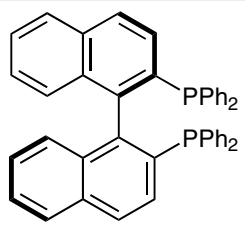

(R)-BINAP

L1<smiles>CC(C)(C)[C@H]1COC(c2ccccc2Pc2ccccc2)=N1</smiles>

L5

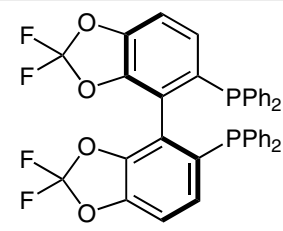

(S)-Difluorphos

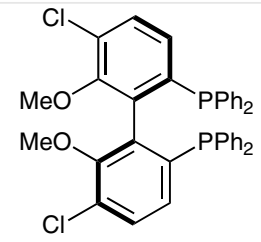

(R)-(-)-Cl-MeO-BIPHEP

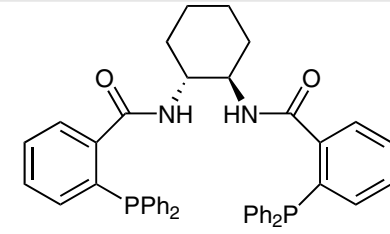

Trost ligand
L3

L4

$\left(S, S_{\text {phos }}, S\right)$-L6, $\mathrm{R}^{1}=i-\mathrm{Pr}$ $\left(S, S_{\text {phos }}, R\right)-\mathrm{L} 7, \mathrm{R}^{1}=i-\mathrm{Pr}$ $\left(S, R_{\text {phos }}, S\right)-\mathrm{L} 8, \mathrm{R}^{1}=i-\mathrm{Pr}$ $\left(S, R_{\text {phos }}, R\right)-\mathrm{L} 9, \mathrm{R}^{1}=i-\mathrm{Pr}$ $\left(S, S_{\text {phos }}, S\right)-$ L10, $\mathrm{R}^{1}=\mathrm{Bn}$ $\left(S, S_{\text {phos }}, S\right)$-L11, $\mathrm{R}^{1}=\mathrm{Ph}$ $\left(S, S_{\text {phos }}, S\right)-\mathrm{L}$ 12, $\mathrm{R}^{1}=t$-Bu $\left(R_{\text {phos }}, R\right)-\mathrm{L}_{13}, \mathrm{R}^{1}=\mathrm{H}$ $\left(S_{\text {phos }}, R\right)-\mathrm{L} 14, \mathrm{R}^{1}=\mathrm{H}$

Figure 2 The structure of ligandsL1-L14 
Table 1 Optimization of Parameters for the Reaction of $\mathbf{1} \mathbf{a}$ with $\mathbf{2}^{\mathrm{a}}$

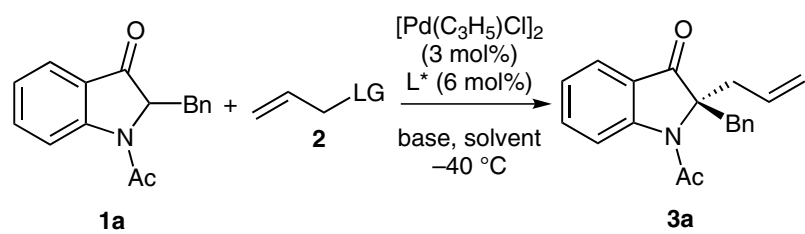

\begin{tabular}{|c|c|c|c|c|c|c|}
\hline Entry & $\mathbf{L}^{*}$ & Base & Solvent & LG (2) & Yield (\%) & ee $(\%)^{c}$ \\
\hline 1 & $\mathbf{L 1}$ & LiHMDS & THF & $\mathrm{OCO}_{2} \mathrm{Me}(\mathbf{2 a})$ & 83 & 38 \\
\hline 2 & L2 & LiHMDS & THF & $\mathrm{OCO}_{2} \mathrm{Me}(\mathbf{2 a})$ & 89 & 55 \\
\hline 3 & L3 & LiHMDS & THF & $\mathrm{OCO}_{2} \mathrm{Me}(\mathbf{2 a})$ & 95 & 40 \\
\hline 4 & L4 & LiHMDS & THF & $\mathrm{OCO}_{2} \mathrm{Me}(\mathbf{2 a})$ & 68 & $-48^{\mathrm{d}}$ \\
\hline 5 & $\mathbf{L} 5$ & LiHMDS & THF & $\mathrm{OCO}_{2} \mathrm{Me}(\mathbf{2 a})$ & 75 & 6 \\
\hline 6 & L2 & LiHMDS & THF & $\mathrm{OCO}_{2} t \mathrm{Bu}(\mathbf{2 b})$ & 90 & 21 \\
\hline 7 & L6 & LiHMDS & THF & $\mathrm{OCO}_{2} \mathrm{Me}(\mathbf{2 a})$ & 99 & 61 \\
\hline 8 & L7 & LiHMDS & THF & $\mathrm{OCO}_{2} \mathrm{Me}(\mathbf{2 a})$ & 98 & 42 \\
\hline 9 & L8 & LiHMDS & THF & $\mathrm{OCO}_{2} \mathrm{Me}(\mathbf{2 a})$ & 97 & $-44^{d}$ \\
\hline 10 & L9 & LiHMDS & THF & $\mathrm{OCO}_{2} \mathrm{Me}(\mathbf{2 a})$ & 90 & $-21^{\mathrm{d}}$ \\
\hline 11 & L10 & LiHMDS & THF & $\mathrm{OCO}_{2} \mathrm{Me}(\mathbf{2 a})$ & 73 & 61 \\
\hline 12 & L11 & LiHMDS & THF & $\mathrm{OCO}_{2} \mathrm{Me}(\mathbf{2 a})$ & 87 & $-16^{d}$ \\
\hline 13 & L12 & LiHMDS & THF & $\mathrm{OCO}_{2} \mathrm{Me}(\mathbf{2 a})$ & 95 & 9 \\
\hline 14 & L13 & LiHMDS & THF & $\mathrm{OCO}_{2} \mathrm{Me}(\mathbf{2 a})$ & 96 & $-31^{d}$ \\
\hline 15 & L14 & LiHMDS & THF & $\mathrm{OCO}_{2} \mathrm{Me}(\mathbf{2 a})$ & 91 & 48 \\
\hline 16 & L6 & LiHMDS & toluene & $\mathrm{OCO}_{2} \mathrm{Me}(\mathbf{2 a})$ & 51 & 42 \\
\hline 17 & L6 & LiHMDS & DME & $\mathrm{OCO}_{2} \mathrm{Me}(\mathbf{2 a})$ & 99 & 76 \\
\hline 18 & L6 & LiHMDS & $\mathrm{Et}_{2} \mathrm{O}$ & $\mathrm{OCO}_{2} \mathrm{Me}(\mathbf{2 a})$ & 99 & 63 \\
\hline 19 & L6 & LiHMDS & $\mathrm{CH}_{2} \mathrm{Cl}_{2}$ & $\mathrm{OCO}_{2} \mathrm{Me}(\mathbf{2 a})$ & 97 & 51 \\
\hline 20 & L6 & NaHMDS & DME & $\mathrm{OCO}_{2} \mathrm{Me}(\mathbf{2 a})$ & 7 & $-16^{d}$ \\
\hline 21 & L6 & KHMDS & DME & $\mathrm{OCO}_{2} \mathrm{Me}(\mathbf{2 a})$ & 10 & 9 \\
\hline 22 & L6 & LDA & DME & $\mathrm{OCO}_{2} \mathrm{Me}(\mathbf{2 a})$ & 6 & 37 \\
\hline $23^{e}$ & L6 & LiHMDS & DME & $\mathrm{OCO}_{2} \mathrm{Me}(\mathbf{2 a})$ & 69 & 72 \\
\hline $24^{f}$ & L6 & LiHMDS & DME & $\mathrm{OCO}_{2} \mathrm{Me}(\mathbf{2 a})$ & 71 & 66 \\
\hline $25^{9}$ & L6 & LiHMDS & DME & $\mathrm{OCO}_{2} \mathrm{Me}(\mathbf{2 a})$ & 76 & 54 \\
\hline
\end{tabular}

a Reaction was carried out at $-40{ }^{\circ} \mathrm{C}$, molar ratio of

$\mathbf{1 a} / \mathbf{2} /\left[\mathrm{Pd}\left(\mathrm{C}_{3} \mathrm{H}_{5}\right) \mathrm{Cl}\right]_{2} / \mathbf{L} /$ base $=100: 150: 3: 6: 120$

${ }^{b}$ Yields of $\mathbf{3 a}$ based on $\mathbf{1 a}$.

' Determined by chiral HPLC analysis.

${ }^{d}$ Reverse sequence of peaks by HPLC.

e $\mathrm{LiCl}$ as additive.

${ }^{f} \mathrm{BnN}(\mathrm{Et})_{3} \mathrm{Cl}$ as additive.

${ }^{9} \mathrm{ZnCl}_{2}$ as additive.

All the experiments were carried out in flame-dried glassware under a dry argon atmosphere. The solvents were purified and dried over appropriate drying agents and distilled under argon prior to use. ${ }^{1} \mathrm{H}$, ${ }^{13} \mathrm{C}$, and ${ }^{19} \mathrm{~F}$ NMR spectra were recorded in $\mathrm{CDCl}_{3}$ using TMS as inter- nal standard with Bruker Avance $300 \mathrm{MHz}, 400 \mathrm{MHz}$, or $600 \mathrm{MHz}$, spectrometers at r.t. HRMS measurements were carried out with a Finnigan MAT 8430 spectrometer.

Synthesis of 2,2-Substituted Indolin-3-ones 3; General Procedure 2-Substituted indolin-3-one $\mathbf{1}(0.1 \mathrm{mmol})$ and DME $(1.0 \mathrm{~mL})$ were added into a dry Schlenk tube. LiHMDS (1.0 M in THF, $0.12 \mathrm{~mL}, 0.12$ $\mathrm{mmol}$ ) was added dropwise and the mixture was stirred for $30 \mathrm{~min}$ at $-40{ }^{\circ} \mathrm{C}$. In a separate flask, $\left[\mathrm{Pd}\left(\mathrm{C}_{3} \mathrm{H}_{5}\right) \mathrm{Cl}\right]_{2}(1.1 \mathrm{mg}, 0.003 \mathrm{mmol})$ and ligand $\left(S, S_{\text {phos }}, S\right)$-SIOCPhox $(\mathbf{L 6} ; 4.1 \mathrm{mg}, 0.006 \mathrm{mmol})$ were dissolved in $\operatorname{DME}(1.0 \mathrm{~mL})$ and the mixture was stirred at r.t. for $30 \mathrm{~min}$, before being added to the above enolate solution at the temperature indicated in Table 2. Allyl methyl carbonate $\mathbf{2 a}(0.15 \mathrm{mmol})$ was then added and the resulting mixture was stirred at the stated temperature. Upon completion (reaction monitored by TLC), the reaction mixture was quenched by sat. aq $\mathrm{NH}_{4} \mathrm{Cl}(5.0 \mathrm{~mL})$ and extracted with EtOAc $(3 \times 10$ $\mathrm{mL})$. The combined organic layer was dried $\left(\mathrm{Na}_{2} \mathrm{SO}_{4}\right)$ and the solvent was removed under reduced pressure. The residue was purified by column chromatography (silica gel; petroleum ether-EtOAc, 10:1) to afford the desired product.

\section{1-Acetyl-2-allyl-2-benzylindolin-3-one (3a)}

Yield: $30.2 \mathrm{mg}$ (99\%); pale-yellow solid; mp $72-75{ }^{\circ} \mathrm{C} ;[\alpha]_{\mathrm{D}}{ }^{24}+10.0(c$ 1.0, $\mathrm{CHCl}_{3}$ ); HPLC [Chiralpak AD-H; $i$-PrOH-hexane (5:95); flow rate: $0.7 \mathrm{~mL} / \mathrm{min} ; \lambda=214 \mathrm{~nm}$ ]: $t_{R}=18.8$ (minor), 15.4 (major) $\mathrm{min}$; ee = $75 \%$.

IR (KBr): 3028, 2962, 2921, 2851, 1711, 1667, 1604, 1469, 1433, 1371 , $1308,1031,751,703 \mathrm{~cm}^{-1}$.

${ }^{1} \mathrm{H}$ NMR $\left(400 \mathrm{MHz}, \mathrm{CDCl}_{3}\right): \delta=7.60(\mathrm{~d}, J=7.2 \mathrm{~Hz}, 1 \mathrm{H}), 7.39(\mathrm{t}, J=$ $7.2 \mathrm{~Hz}, 1 \mathrm{H}), 7.12(\mathrm{~d}, J=5.6 \mathrm{~Hz}, 1 \mathrm{H}), 6.99-6.93(\mathrm{~m}, 6 \mathrm{H}), 5.36-5.25(\mathrm{~m}$, $1 \mathrm{H}), 5.04(\mathrm{~d}, J=16.8 \mathrm{~Hz}, 1 \mathrm{H}), 4.83(\mathrm{~d}, J=9.6 \mathrm{~Hz}, 1 \mathrm{H}), 3.74(\mathrm{~d}, J=$ $12.4 \mathrm{~Hz}, 1 \mathrm{H}), 3.36(\mathrm{br}, 1 \mathrm{H}), 3.22(\mathrm{~d}, J=13.2 \mathrm{~Hz}, 1 \mathrm{H}), 2.82(\mathrm{br}, 1 \mathrm{H})$, $2.44(\mathrm{~s}, 3 \mathrm{H})$.

${ }^{13} \mathrm{C}$ NMR $\left(100 \mathrm{MHz}, \mathrm{CDCl}_{3}\right): \delta=202.0,168.6,152.5,136.7,135.1$, $131.2,129.4,127.8,126.7,125.3,124.1,123.2,119.4,114.7,77.0,41.6$, 40.4, 27.1

MS (EI): $m / z(\%)=305(39)[\mathrm{M}]^{+}, 264(21), 222(61), 214(17), 172$ (100), 144 (10), 130 (10), 115 (12), 91 (50), 77 (13), 43 (30).

HRMS (ESI): $m / z[\mathrm{M}+\mathrm{H}]^{+}$calcd for $\mathrm{C}_{20} \mathrm{H}_{20} \mathrm{NO}_{2}$ : 306.1489; found: 306.1495.

\section{1-Acetyl-2-allyl-2-methylindolin-3-one (3b)}

Yield: $14.2 \mathrm{mg}$ (62\%); pale-yellow solid; $\mathrm{mp} 90-91{ }^{\circ} \mathrm{C} ;[\alpha]_{\mathrm{D}}{ }^{27}+52.9(\mathrm{c}$ 1.00, $\mathrm{CHCl}_{3}$ ); HPLC [Chiralpak AD-H; $i$-PrOH-hexane (2:98); flow rate: $0.7 \mathrm{~mL} / \mathrm{min} ; \lambda=214 \mathrm{~nm}$ ]: $t_{R}=18.7$ (minor), 21.1 (major) min; ee $=88 \%$.

IR (KBr): 3072, 1703, 1667, 1641, 1608, 1471, 1432, 1371, 1343, 1310, $1191,1100,930,750 \mathrm{~cm}^{-1}$.

${ }^{1} \mathrm{H}$ NMR (400 MHz, $\left.\mathrm{CDCl}_{3}\right): \delta=8.32(\mathrm{br}, 1 \mathrm{H}), 7.78(\mathrm{~d}, J=7.6 \mathrm{~Hz}, 1 \mathrm{H})$, $7.65(\mathrm{dt}, J=7.8,1.2 \mathrm{~Hz}, 1 \mathrm{H}), 7.20(\mathrm{t}, J=7.6 \mathrm{~Hz}, 1 \mathrm{H}), 5.33-5.23(\mathrm{~m}$, $1 \mathrm{H}), 5.02(\mathrm{~d}, J=16.8 \mathrm{~Hz}, 1 \mathrm{H}), 4.86(\mathrm{~d}, J=9.6 \mathrm{~Hz}, 1 \mathrm{H}), 3.10(\mathrm{br}, 1 \mathrm{H})$, 2.76 (br, $1 \mathrm{H}), 2.51(\mathrm{~s}, 3 \mathrm{H}), 1.60$ (s, $3 \mathrm{H})$.

${ }^{13} \mathrm{C}$ NMR $\left(100 \mathrm{MHz}, \mathrm{CDCl}_{3}\right): \delta=201.8,168.7,152.8,137.4,131.1$, 124.7, 123.9, 119.5, 117.1, 116.1, 72.0, 40.9, 26.7, 22.8.

MS (EI): $m / z(\%)=229(14)[\mathrm{M}]^{+}, 188(37), 147(15), 146(100), 144(9)$, 117 (10), 91 (10), 77 (15).

HRMS (ESI): $m / z[\mathrm{M}+\mathrm{H}]^{+}$calcd for $\mathrm{C}_{14} \mathrm{H}_{16} \mathrm{NO}_{2}$ : 230.1176; found: 230.1182 . 
Table 2 Substrate Scope for Palladium-Catalyzed AAA<smiles>[R7]c1ccc2c(c1)N([CH])C([R])C2=O</smiles>

1<smiles>C=CCOC(=O)OC</smiles>

2a
$\left(S, S_{\text {phos }}, S\right)$-L6 (6 mol\%)

$\left[\mathrm{Pd}\left(\mathrm{C}_{3} \mathrm{H}_{5}\right) \mathrm{Cl}\right]_{2}(3 \mathrm{~mol} \%)$

LiHMDS, DME, $-40^{\circ} \mathrm{C}$<smiles>[R7]C1(CC=C)C(=O)c2ccc([Tl])cc2N1C(=O)O</smiles>

3
1k: $\mathrm{R}^{1}=\mathrm{H}, \mathrm{R}^{2}=p-(i-\mathrm{Pr}) \mathrm{C}_{6} \mathrm{H}_{4} \mathrm{CH}_{2}$
1l: $\mathrm{R}^{1}=\mathrm{H}, \mathrm{R}^{2}=o-\mathrm{NCC}_{6} \mathrm{H}_{4} \mathrm{CH}_{2}$
1m: $\mathrm{R}^{1}=\mathrm{H}, \mathrm{R}^{2}=m-\mathrm{MeC}_{6} \mathrm{H}_{4} \mathrm{CH}_{2}$
1n: $R^{1}=H, R^{2}=$ furan-2-ylmethyl
1a: $R^{1}=H, R^{2}=B n \quad$ 1f: $R^{1}=F, R^{2}=M e$
1b: $R^{1}=H, R^{2}=M e$ 1g: $R^{1}=H, R^{2}=P h$
1c: $\mathrm{R}^{1}=\mathrm{Cl}, \mathrm{R}^{2}=\mathrm{Bn}$ 1h: $\mathrm{R}^{1}=\mathrm{H}, \mathrm{R}^{2}=o-\mathrm{BrC}_{6} \mathrm{H}_{4}$
1d: $R^{1}=\mathrm{Cl}, \mathrm{R}^{2}=$ Me 1i: $\mathrm{R}^{1}=\mathrm{H}, \mathrm{R}^{2}=\mathrm{Et}$
1e: $R^{1}=F, R^{2}=B n \quad 1 j: R^{1}=H, R^{2}=o-B_{C} C_{6} H_{4} C H_{2}$

\begin{tabular}{|c|c|c|c|c|}
\hline Entry & Substrate & Product & Yield (\%) & ee $(\%)^{c}$ \\
\hline 1 & $1 a$ & $3 a$ & 99 & 75 \\
\hline 2 & $1 b$ & $3 b$ & 62 & 88 \\
\hline 3 & 1c & $3 c$ & 80 & 74 \\
\hline 4 & 1d & $3 d$ & 70 & 86 \\
\hline 5 & $1 e$ & $3 e$ & 76 & 86 \\
\hline 6 & $1 f$ & $3 f$ & 66 & 86 \\
\hline $7^{d}$ & $1 \mathrm{~g}$ & $3 g$ & 86 & 92 \\
\hline $8^{d}$ & $1 \mathrm{~h}$ & $3 \mathrm{~h}$ & 92 & 87 \\
\hline 9 & $1 \mathbf{i}$ & $3 \mathbf{i}$ & 60 & 78 \\
\hline 10 & $1 \mathrm{j}$ & $3 \mathbf{j}$ & 83 & 84 \\
\hline 11 & $1 \mathrm{k}$ & $3 k$ & 81 & 75 \\
\hline $12^{\mathrm{e}}$ & 11 & 31 & 93 & 84 \\
\hline $13^{e}$ & $1 \mathrm{~m}$ & $3 m$ & 83 & 68 \\
\hline 14 & 1n & $3 n$ & 80 & 76 \\
\hline
\end{tabular}

a Reaction was carried out at $-40{ }^{\circ} \mathrm{C}$, molar ratio of $\mathbf{1} / \mathbf{2 a} /\left[\mathrm{Pd}\left(\mathrm{C}_{3} \mathrm{H}_{5}\right) \mathrm{Cl}\right]_{2} / \mathbf{L 6} /$ base $=100: 150: 3: 6: 120$.

b Yield of $\mathbf{3}$ based on $\mathbf{1}$

'Determined by chiral HPLC analysis.

d Reaction was carried out at $15^{\circ} \mathrm{C}$.

e Reaction was carried out at $0{ }^{\circ} \mathrm{C}$.

\section{1-Acetyl-2-allyl-2-benzyl-6-chloroindolin-3-one (3c)}

Yield: $27.1 \mathrm{mg}$ (80\%); pale-yellow solid; $\mathrm{mp} 50-52^{\circ} \mathrm{C} ;[\alpha]_{\mathrm{D}}{ }^{24}+43.2(\mathrm{c}$ 1.04, $\mathrm{CHCl}_{3}$ ); HPLC [Chiralpak AD-H; $i$-PrOH-hexane (5:95); flow rate: $0.7 \mathrm{~mL} / \mathrm{min} ; \lambda=214 \mathrm{~nm}$ ]: $t_{R}=11.7$ (minor), 11.0 (major) $\mathrm{min}$; ee $=74 \%$.

IR (KBr): 3083, 2956, 2925, 2854, 1716, 1673, 1603, 1574, 1428, 1370 , $1312,1272,1083,992,702 \mathrm{~cm}^{-1}$.

${ }^{1} \mathrm{H}$ NMR $\left(400 \mathrm{MHz}, \mathrm{CDCl}_{3}\right): \delta=7.52(\mathrm{~d}, J=7.2 \mathrm{~Hz}, 1 \mathrm{H}), 7.13(\mathrm{~s}, 1 \mathrm{H})$, 7.00-6.92 (m, $6 \mathrm{H}), 5.34-5.24(\mathrm{~m}, 1 \mathrm{H}), 5.05(\mathrm{~d}, J=17.2 \mathrm{~Hz}, 1 \mathrm{H}), 4.87$ (d, $J=9.2 \mathrm{~Hz}, 1 \mathrm{H}), 3.72(\mathrm{br}, 1 \mathrm{H}), 3.34-3.22(\mathrm{~m}, 2 \mathrm{H}), 2.81$ (br, $1 \mathrm{H})$, $2.44(\mathrm{~s}, 3 \mathrm{H})$.

${ }^{13} \mathrm{C}$ NMR $\left(100 \mathrm{MHz}, \mathrm{CDCl}_{3}\right): \delta=200.6,168.5,153.0,143.3,134.9$, 130.9, 129.4, 128.1, 127.0, 125.0, 123.9, 119.8, 115.1, 77.8, 41.6, 40.5, 27.1.

MS (EI): $m / z(\%)=341(39)[\mathrm{M}]^{+}, 339(95)[\mathrm{M}]^{+}, 298(45), 258$ (67), 256 (100), 208 (97), 207 (97), 206 (100), 164 (26), 143 (33), 115 (29), 91 (96).
HRMS (ESI): $m / z[\mathrm{M}+\mathrm{H}]^{+}$calcd for $\mathrm{C}_{20} \mathrm{H}_{19} \mathrm{ClNO}_{2}$ : 340.1099; found: 340.1098 .

\section{1-Acetyl-2-allyl-6-chloro-2-methylindolin-3-one (3d)}

Yield: $18.4 \mathrm{mg}$ (70\%); slightly yellow oil; $[\alpha]_{\mathrm{D}}{ }^{20}+51.3$ (c 1.05, $\mathrm{CHCl}_{3}$ ); HPLC [Chiralpak AD-H; $i$-PrOH-hexane (3:97); flow rate: $0.7 \mathrm{~mL} / \mathrm{min}$; $\lambda=214 \mathrm{~nm}$ ]: $t_{R}=12.8$ (minor), 13.5 (major) $\mathrm{min}$; ee $=86 \%$.

IR (KBr): 3080, 2979, 2928, 2855, 1717, 1672, 1602, 1574, 1425, 1368 , $1341,1269,1183,979,925,826 \mathrm{~cm}^{-1}$.

${ }^{1} \mathrm{H}$ NMR (400 MHz, $\left.\mathrm{CDCl}_{3}\right): \delta=8.05(\mathrm{br}, 1 \mathrm{H}), 7.66(\mathrm{~d}, J=8.4 \mathrm{~Hz}, 1 \mathrm{H})$, $7.15(\mathrm{dd} J=8.0,1.2 \mathrm{~Hz}, 1 \mathrm{H}), 5.29-5.18(\mathrm{~m}, 1 \mathrm{H}), 5.00(\mathrm{~d}, J=16.8 \mathrm{~Hz}$, $1 \mathrm{H}), 4.86$ (d, $J=10.4 \mathrm{~Hz}, 1 \mathrm{H}), 3.00(\mathrm{br}, 1 \mathrm{H}), 2.73$ (br, $1 \mathrm{H}), 2.47$ (s, $3 \mathrm{H}), 1.56$ (s, $3 \mathrm{H})$.

${ }^{13} \mathrm{C}$ NMR $\left(100 \mathrm{MHz}, \mathrm{CDCl}_{3}\right): \delta=200.1,168.7,153.1,143.8,130.6$, 125.2, 124.6, 121.8, 119.8, 117.8, 71.8, 41.1, 26.1, 22.9.

MS (EI): $m / z(\%)=265(6)[\mathrm{M}]^{+}, 263(20)[\mathrm{M}]^{+}, 224(11), 222(38), 182$ (34), 180 (100), 117 (11), 110 (14), 75 (20), 43 (55). 
HRMS (ESI): $m / z[\mathrm{M}+\mathrm{H}]^{+}$calcd for $\mathrm{C}_{14} \mathrm{H}_{15} \mathrm{ClNO}_{2}$ : 264.0786; found: 264.0780 .

\section{1-Acetyl-2-allyl-2-benzyl-6-fluoroindolin-3-one (3e)}

Yield: $24.5 \mathrm{mg}$ (76\%); pale-solid; $\mathrm{mp} 81-83{ }^{\circ} \mathrm{C} ;[\alpha]_{D}{ }^{20}+17.2$ (c 1.12, $\mathrm{CHCl}_{3}$ ); HPLC [Chiralpak AD; $i$-PrOH-hexane (5:95); flow rate: 0.6 $\mathrm{mL} / \mathrm{min} ; \lambda=214 \mathrm{~nm}$ ]: $t_{R}=15.0$ (minor), 13.9 (major) $\mathrm{min}$; ee $=86 \%$. IR (KBr): 3084, 3032, 2931, 1716, 1677, 1619, 1589, 1488, 1436, 1372 , $1275,1184,924,703 \mathrm{~cm}^{-1}$.

${ }^{1} \mathrm{H}$ NMR $\left(400 \mathrm{MHz}, \mathrm{CDCl}_{3}\right): \delta=7.62(\mathrm{t}, J=4.8 \mathrm{~Hz}, 1 \mathrm{H}), 7.03-7.00(\mathrm{~m}$, $3 \mathrm{H}), 6.94-6.92(\mathrm{~m}, 2 \mathrm{H}), 6.81(\mathrm{br}, 1 \mathrm{H}), 6.72(\mathrm{dt}, J=8.1,2.0 \mathrm{~Hz}, 1 \mathrm{H})$, 5.37-5.26 (m, $1 \mathrm{H}), 5.07$ (d, $J=17.2 \mathrm{~Hz}, 1 \mathrm{H}), 4.89$ (d, $J=8.8 \mathrm{~Hz}, 1 \mathrm{H})$, 3.73 (br, $1 \mathrm{H}$ ), 3.36-3.23 (m, $2 \mathrm{H}$ ), 2.83 (br, $1 \mathrm{H}), 2.44$ (s, $3 \mathrm{H}$ ).

${ }^{13} \mathrm{C} \mathrm{NMR}\left(100 \mathrm{MHz}, \mathrm{CDCl}_{3}\right): \delta=200.2,168.5,168.2\left(\mathrm{~d}, J_{\mathrm{C}-\mathrm{F}}=255.0 \mathrm{~Hz}\right)$, $153.9,134.9,131.0,129.4,128.0,126.9,126.3,121.9,119.7,111.4$ (d, $\left.J_{\mathrm{C}-\mathrm{F}}=22.6 \mathrm{~Hz}\right), 102.6\left(\mathrm{~d}, J_{\mathrm{C}-\mathrm{F}}=27.1 \mathrm{~Hz}\right), 77.9,41.6,40.4,27.0$.

${ }^{19} \mathrm{~F}$ NMR (376 MHz, $\left.\mathrm{CDCl}_{3}\right): \delta=-97.44$.

MS (EI): $m / z(\%)=323(55)[\mathrm{M}]^{+}, 282(20), 240$ (95), 232 (30), 191 (28), 190 (100), 162 (19), 148 (17), 91 (62).

HRMS (ESI): $m / z[\mathrm{M}+\mathrm{H}]^{+}$calcd for $\mathrm{C}_{20} \mathrm{H}_{19} \mathrm{FNO}_{2}$ : 324.1394; found: 324.1381 .

\section{1-Acetyl-2-allyl-6-fluoro-2-methylindolin-3-one (3f)}

Yield: $16.3 \mathrm{mg}(66 \%)$; slightly yellow oil; $[\alpha]_{\mathrm{D}}{ }^{20.7}+63.0\left(\right.$ c $\left.1.00, \mathrm{CHCl}_{3}\right)$; HPLC [Chiralpak AD-H;i-PrOH-hexane (3:97); flow rate: $0.7 \mathrm{~mL} / \mathrm{min}$; $\lambda=214 \mathrm{~nm}$ ]: $t_{R}=13.6$ (minor), 14.7 (major) min; ee $=86 \%$.

IR (KBr): 3082, 2980, 2931, 2856, 1717, 1673, 1618, 1589, 1437, 1370 , $1345,1267,1192,987,924,843 \mathrm{~cm}^{-1}$.

${ }^{1} \mathrm{H}$ NMR $\left(400 \mathrm{MHz}, \mathrm{CDCl}_{3}\right): \delta=7.86(\mathrm{br}, 1 \mathrm{H}), 7.73(\mathrm{dd}, J=8.0,6.4 \mathrm{~Hz}$, $1 \mathrm{H}), 6.87(\mathrm{t}, J=8.4 \mathrm{~Hz}, 1 \mathrm{H}), 5.29-5.18(\mathrm{~m}, 1 \mathrm{H}), 4.99(\mathrm{~d}, J=16.8 \mathrm{~Hz}$, $1 \mathrm{H}), 4.85(\mathrm{~d}, J=10.0 \mathrm{~Hz}, 1 \mathrm{H}), 2.99(\mathrm{br}, 1 \mathrm{H}), 2.74(\mathrm{br}, 1 \mathrm{H}), 2.45(\mathrm{~s}$, $3 \mathrm{H}), 1.56(\mathrm{~s}, 3 \mathrm{H})$.

${ }^{13} \mathrm{C}$ NMR $\left(75 \mathrm{MHz}, \mathrm{CDCl}_{3}\right): \delta=199.6,170.3,168.6,166.9,154.0,130.5$, $126.3,119.6,112.0\left(\mathrm{~d}, J_{\mathrm{C}-\mathrm{F}}=23.9 \mathrm{~Hz}\right), 105.1,72.1,41.0,25.9,22.8$.

${ }^{19} \mathrm{~F} \mathrm{NMR}\left(376 \mathrm{MHz}, \mathrm{CDCl}_{3}\right): \delta=-97.04$.

MS (EI): $m / z(\%)=247(41)[\mathrm{M}]^{+}, 206$ (89), 165 (44), 164 (100), 162 (21), 135 (24), 109 (22), 95 (25), 94 (36).

HRMS (ESI): $m / z[\mathrm{M}+\mathrm{H}]^{+}$calcd for $\mathrm{C}_{14} \mathrm{H}_{15} \mathrm{FNO}_{2}$ : 248.1081; found: 248.1072 .

\section{1-Acetyl-2-allyl-2-phenylindolin-3-one (3g)}

Yield: $25.0 \mathrm{mg}$ (86\%); pale solid; $\mathrm{mp} 121-123{ }^{\circ} \mathrm{C} ;[\alpha]_{\mathrm{D}}^{25.5}+338.9(\mathrm{c}$ $0.70, \mathrm{CHCl}_{3}$ ); HPLC [Chiralpak AD; $i$-PrOH-hexane (5:95); flow rate: $0.6 \mathrm{~mL} / \mathrm{min} ; \lambda=214 \mathrm{~nm}$ ]: $t_{R}=23.2$ (minor), 22.0 (major) $\mathrm{min}$; ee = $92 \%$.

IR (KBr): 3067, 2919, 2852, 1721, 1670, 1606, 1460, 1370, 1337, 1292 , $1149,914,756,701 \mathrm{~cm}^{-1}$.

${ }^{1} \mathrm{H}$ NMR $\left(400 \mathrm{MHz}, \mathrm{CDCl}_{3}\right): \delta=8.81(\mathrm{~d}, J=8.0 \mathrm{~Hz}, 1 \mathrm{H}), 7.76-7.72(\mathrm{~m}$, $2 \mathrm{H}), 7.38-7.32(\mathrm{~m}, 3 \mathrm{H}), 7.28-7.24(\mathrm{~m}, 3 \mathrm{H}), 5.46-5.36(\mathrm{~m}, 1 \mathrm{H}), 5.19$ $(\mathrm{d}, J=16.8 \mathrm{~Hz}, 1 \mathrm{H}), 5.02(\mathrm{~d}, J=10.0 \mathrm{~Hz}, 1 \mathrm{H}), 3.66(\mathrm{dd}, J=13.2,4.8 \mathrm{~Hz}$, $1 \mathrm{H}), 3.15(\mathrm{dd}, J=13.2,8.0 \mathrm{~Hz}, 1 \mathrm{H}), 2.00(\mathrm{~s}, 3 \mathrm{H})$.

${ }^{13} \mathrm{C}$ NMR $\left(100 \mathrm{MHz}, \mathrm{CDCl}_{3}\right): \delta=197.7,170.1,154.6,137.8,137.6$, 129.6, 129.5, 128.5, 125.1, 124.8, 124.7, 122.3, 120.6, 118.9, 74.7, 40.2, 25.1.

MS (EI): $m / z(\%)=291(64)[\mathrm{M}]^{+}, 250$ (96), 220 (21), 209 (91), 208 (100), 180 (31), 152 (27), 77 (42).
HRMS (ESI): $m / z[M+\mathrm{H}]^{+}$calcd for $\mathrm{C}_{19} \mathrm{H}_{18} \mathrm{NO}_{2}$ : 292.1332; found: 292.1334 .

\section{1-Acetyl-2-allyl-2-(2-bromophenyl)indolin-3-one (3h)}

Yield: $33.9 \mathrm{mg}$ (92\%); pale-yellow solid; $\mathrm{mp} 120-122^{\circ} \mathrm{C} ;[\alpha]_{\mathrm{D}} 25.5$ +157.4 (c 1.09, $\mathrm{CHCl}_{3}$ ); HPLC [Chiralpak AD-H; $i$-PrOH-hexane (20:80); flow rate: $0.7 \mathrm{~mL} / \mathrm{min} ; \lambda=214 \mathrm{~nm}$ ]: $t_{R}=14.9$ (minor), 19.8 (major) $\mathrm{min}$; ee $=87 \%$.

IR (KBr): 3077, 2923, 2850, 1718, 1668, 1607, 1588, 1460, 1369, 1339 , $1291,1197,1004,911,750 \mathrm{~cm}^{-1}$.

${ }^{1} \mathrm{H} \mathrm{NMR}\left(300 \mathrm{MHz}, \mathrm{CDCl}_{3}\right): \delta=8.62(\mathrm{br}, 1 \mathrm{H}), 7.76(\mathrm{~m}, 3 \mathrm{H}), 7.50$ (d, $J=$ $7.5 \mathrm{~Hz}, 1 \mathrm{H}), 7.41(\mathrm{t}, J=7.5 \mathrm{~Hz}, 1 \mathrm{H}), 7.23-7.15(\mathrm{~m}, 2 \mathrm{H}), 5.41-5.25(\mathrm{~m}$ $1 \mathrm{H}), 5.08(\mathrm{~d}, J=15.9 \mathrm{~Hz}, 1 \mathrm{H}), 4.88(\mathrm{~d}, J=10.2 \mathrm{~Hz}, 1 \mathrm{H}), 3.37(\mathrm{br}, 1 \mathrm{H})$, 3.23 (br, $1 \mathrm{H}), 1.95$ (br, $3 \mathrm{H}$ ).

${ }^{13} \mathrm{C}$ NMR $\left(75 \mathrm{MHz}, \mathrm{CDCl}_{3}\right): \delta=198.3,168.5,154.3,137.2,135.2,130.5$, 130.0, 129.6, 127.8, 125.7, 124.2, 123.3, 121.6, 120.8, 119.0, 74.4, 41.8, 25.1 .

MS (EI): $m / z(\%)=371(5)[\mathrm{M}]^{+}, 369(6)[\mathrm{M}]^{+}, 330$ (17), 328 (23), 290 (53), 288 (93), 286 (95), 207 (100), 178 (18), 151 (18), 102 (18), 76 (36).

HRMS (ESI): $m / z$ [M $+\mathrm{H}]^{+}$calcd for $\mathrm{C}_{19} \mathrm{H}_{17} \mathrm{BrNO}_{2}$ : 370.0437; found: 370.0437.

\section{1-Acetyl-2-allyl-2-ethylindolin-3-one (3i)}

Yield: $14.6 \mathrm{mg}(60 \%)$; slightly yellow oil; $[\alpha]_{\mathrm{D}}^{24.5}+6.3\left(\right.$ c $\left.1.00, \mathrm{CHCl}_{3}\right)$; HPLC [Chiralpak AD; $i$-PrOH-hexane (5:95); flow rate: $0.6 \mathrm{~mL} / \mathrm{min}$; $\lambda=214 \mathrm{~nm}$ ): $t_{R}=13.6$ (minor), 14.1 (major) $\min$; ee $=78 \%$.

IR (KBr): 3079, 2971, 2934, 2878, 1714, 1667, 1609, 1460, 1370, 1295 , $920,754 \mathrm{~cm}^{-1}$.

${ }^{1} \mathrm{H}$ NMR $\left(400 \mathrm{MHz}, \mathrm{CDCl}_{3}\right): \delta=8.67(\mathrm{br}, 1 \mathrm{H}), 7.76(\mathrm{~d}, J=8.0 \mathrm{~Hz}, 1 \mathrm{H})$, $7.65(\mathrm{td}, J=8.0,1.2 \mathrm{~Hz}, 1 \mathrm{H}), 7.19(\mathrm{t}, J=7.6 \mathrm{~Hz}, 1 \mathrm{H}), 5.33-5.23(\mathrm{~m}$, $1 \mathrm{H}), 5.01(\mathrm{~d}, J=16.8 \mathrm{~Hz}, 1 \mathrm{H}), 4.85(\mathrm{~d}, J=10.0 \mathrm{~Hz}, 1 \mathrm{H}), 3.14(\mathrm{br}, 1 \mathrm{H})$, 2.72-2.52 (m, $5 \mathrm{H}), 2.04(\mathrm{br}, 1 \mathrm{H}), 0.56(\mathrm{t}, J=7.6 \mathrm{~Hz}, 3 \mathrm{H})$.

${ }^{13} \mathrm{C}$ NMR $\left(150 \mathrm{MHz}, \mathrm{CDCl}_{3}\right): \delta=202.2,168.3,152.9,137.2,131.2$, $125.1,124.1,123.6,119.3,115.2,74.4,40.6,29.6,26.9,7.8$.

MS (EI): $m / z(\%)=243(20)[M]^{+}, 202(50), 161$ (18), 160 (100), 132 (26), 130 (19), 117 (15), 77 (18).

HRMS (ESI): $m / z[M+\mathrm{H}]^{+}$calcd for $\mathrm{C}_{15} \mathrm{H}_{18} \mathrm{NO}_{2}$ : 244.1332; found: 244.1333 .

\section{1-Acetyl-2-allyl-2-(2-bromobenzyl)indolin-3-one (3j)}

Yield: $31.8 \mathrm{mg}$ (83\%); pale-yellow solid; $\mathrm{mp} 55-57^{\circ} \mathrm{C} ;[\alpha]_{\mathrm{D}}^{24.5}-20.2(\mathrm{c}$ 1.00, $\mathrm{CHCl}_{3}$ ); HPLC [Chiralpak AD-H; $i-\mathrm{PrOH}-$ hexane (5:95); flow rate: $0.7 \mathrm{~mL} / \mathrm{min} ; \lambda=214 \mathrm{~nm}$ ]: $t_{R}=22.0$ (minor), 17.3 (major) min; ee $=84 \%$.

IR (KBr): 3077, 2929, 1715, 1668, 1608, 1470, 1435, 1369, 1298, 1024, $926,751 \mathrm{~cm}^{-1}$.

${ }^{1} \mathrm{H}$ NMR $\left(300 \mathrm{MHz}, \mathrm{CDCl}_{3}\right): \delta=8.50(\mathrm{br}, 1 \mathrm{H}), 7.61(\mathrm{~d}, J=7.5 \mathrm{~Hz}, 1 \mathrm{H})$, $7.49(\mathrm{t}, J=7.8 \mathrm{~Hz}, 1 \mathrm{H}), 7.30(\mathrm{~d}, J=8.1 \mathrm{~Hz}, 1 \mathrm{H}), 7.06-6.96(\mathrm{~m}, 3 \mathrm{H})$, $6.86(\mathrm{t}, J=8.1 \mathrm{~Hz}, 1 \mathrm{H}), 5.37-5.24(\mathrm{~m}, 1 \mathrm{H}), 5.08(\mathrm{~d}, J=16.2 \mathrm{~Hz}, 1 \mathrm{H})$, $4.89(\mathrm{~d}, J=9.9 \mathrm{~Hz}, 1 \mathrm{H}), 3.85(\mathrm{br}, 1 \mathrm{H}), 3.52(\mathrm{br}, 1 \mathrm{H}), 3.43(\mathrm{~d}, J=$ $14.1 \mathrm{~Hz}, 1 \mathrm{H}), 2.91$ (br, $1 \mathrm{H}), 2.50$ (s, $3 \mathrm{H})$.

${ }^{13} \mathrm{C}$ NMR $\left(150 \mathrm{MHz}, \mathrm{CDCl}_{3}\right): \delta=200.6,168.5,152.2,136.6,135.0$, $132.8,132.1,131.2,128.3,126.7,125.2,125.0,124.3,123.3,119.6$, $115.0,76.3,40.7,39.9,27.0$. 
MS (EI): $m / z(\%)=385(36)[\mathrm{M}]^{+}, 383(37)[\mathrm{M}]^{+}, 344(24), 342(27), 302$ (69), 300 (72), 220 (52), 214 (98), 173 (84), 172 (100), 144 (33), 130 (28), 115 (25), 90 (25).

HRMS (ESI): $m / z[\mathrm{M}+\mathrm{H}]^{+}$calcd for $\mathrm{C}_{20} \mathrm{H}_{19} \mathrm{BrNO}_{2}$ : 384.0594; found: 384.0593 .

\section{1-Acetyl-2-allyl-2-(4-isopropylbenzyl)indolin-3-one (3k)}

Yield: $28.1 \mathrm{mg}(81 \%)$; slightly yellow oil; $[\alpha]_{\mathrm{D}}^{24.6}+6.4\left(\right.$ c $\left.0.92, \mathrm{CHCl}_{3}\right)$; HPLC [Chiralpak AD-H; $i$-PrOH-hexane (5:95); flow rate: $0.7 \mathrm{~mL} / \mathrm{min}$; $\lambda=214 \mathrm{~nm}$ ]: $t_{R}=15.4$ (minor), 12.5 (major) $\min$; ee $=75 \%$.

IR (KBr): 3080, 2960, 2927, 2871, 1716, 1670, 1609, 1470, 1372, 1311, $1290,924,795 \mathrm{~cm}^{-1}$.

${ }^{1} \mathrm{H}$ NMR $\left(400 \mathrm{MHz}, \mathrm{CDCl}_{3}\right): \delta=7.61(\mathrm{~d}, J=7.6 \mathrm{~Hz}, 1 \mathrm{H}), 7.41(\mathrm{t}, J=$ $8.0 \mathrm{~Hz}, 1 \mathrm{H}), 7.13(\mathrm{~d}, J=6.4 \mathrm{~Hz}, 1 \mathrm{H}), 7.00(\mathrm{t}, J=7.6 \mathrm{~Hz}, 1 \mathrm{H}), 6.86-6.81$ (m, $4 \mathrm{H}), 5.38-5.27(\mathrm{~m}, 1 \mathrm{H}), 5.06(\mathrm{~d}, J=17.2 \mathrm{~Hz}, 1 \mathrm{H}), 4.86(\mathrm{~d}, J=$ $10.0 \mathrm{~Hz}, 1 \mathrm{H}), 3.72(\mathrm{~d}, J=12.4 \mathrm{~Hz}, 1 \mathrm{H}), 3.40-3.33(\mathrm{~m}, 1 \mathrm{H}), 3.20(\mathrm{~d}, J=$ $13.2 \mathrm{~Hz}, 1 \mathrm{H}), 2.85-2.80(\mathrm{~m}, 1 \mathrm{H}), 2.69-2.61(\mathrm{~m}, 1 \mathrm{H}), 2.46(\mathrm{~s}, 3 \mathrm{H})$, $1.05(\mathrm{~d}, J=2.4 \mathrm{~Hz}, 3 \mathrm{H}), 1.03(\mathrm{~d}, J=2.4 \mathrm{~Hz}, 3 \mathrm{H})$.

${ }^{13} \mathrm{C} \mathrm{NMR}\left(75 \mathrm{MHz}, \mathrm{CDCl}_{3}\right): \delta=202.2,168.7,152.6,147.3,136.6,132.4$, 131.4, 129.4, 125.8, 125.5, 124.3, 123.1, 119.4, 114.9, 74.3, 41.3, 40.3, 33.6, 27.2, 23.9 .

MS (EI): $m / z(\%)=347(15)[\mathrm{M}]^{+}, 264$ (21), 214 (12), 172 (100), 133 (38), 117 (18), 105 (11), 91 (11).

HRMS (ESI): $m / z[M+\mathrm{H}]^{+}$calcd for $\mathrm{C}_{23} \mathrm{H}_{26} \mathrm{NO}_{2}$ : 348.1958; found: 348.1959 .

\section{2-[(1-Acetyl-2-allyl-3-oxoindolin-2-yl)methyl]benzonitrile (31)}

Yield: $30.7 \mathrm{mg}$ (93\%); pale-solid; mp $83-85{ }^{\circ} \mathrm{C} ;[\alpha]_{\mathrm{D}}^{24.7}-14.3$ (c 1.35, $\mathrm{CHCl}_{3}$ ); HPLC [Chiralpak AD-H; $i$-PrOH-hexane (8:92); flow rate: 1.0 $\mathrm{mL} / \mathrm{min} ; \lambda=214 \mathrm{~nm}$ ]: $t_{R}=22.9$ (minor), 20.6 (major) $\mathrm{min}$; ee $=84 \%$.

IR (KBr): 3080, 3025, 2937, 2854, 2225, 1707, 1674, 1608, 1470, 1368, $1272,933,910,753 \mathrm{~cm}^{-1}$.

${ }^{1} \mathrm{H}$ NMR (400 MHz, $\left.\mathrm{CDCl}_{3}\right): \delta=7.59(\mathrm{~d}, J=8.0 \mathrm{~Hz}, 1 \mathrm{H}), 7.53(\mathrm{t}, J=$ $8.0 \mathrm{~Hz}, 1 \mathrm{H}), 7.41(\mathrm{~d}, J=7.2 \mathrm{~Hz}, 1 \mathrm{H}), 7.35(\mathrm{br}, 1 \mathrm{H}), 7.28(\mathrm{t}, J=7.6 \mathrm{~Hz}$, $1 \mathrm{H}), 7.14(\mathrm{t}, J=7.2 \mathrm{~Hz}, 2 \mathrm{H}), 7.05(\mathrm{t}, J=7.2 \mathrm{~Hz}, 1 \mathrm{H}), 5.36-5.26(\mathrm{~m}$, $1 \mathrm{H}), 5.10(\mathrm{~d}, J=17.2 \mathrm{~Hz}, 1 \mathrm{H}), 4.91(\mathrm{~d}, J=9.6 \mathrm{~Hz}, 1 \mathrm{H}), 3.98(\mathrm{~d}, J=$ $10.8 \mathrm{~Hz}, 1 \mathrm{H}), 3.52-3.43$ (m, $2 \mathrm{H}), 2.90$ (br, $1 \mathrm{H}), 2.56$ (s, $3 \mathrm{H})$.

${ }^{13} \mathrm{C}$ NMR $\left(100 \mathrm{MHz}, \mathrm{CDCl}_{3}\right): \delta=200.4,168.8,152.5,138.9,137.1$, $133.1,132.1,131.4,130.9,127.5,124.8,124.5,123.5,120.1,118.1$, 115.2, 113.5, 76.1, 40.0, 39.7, 27.1 .

MS (EI): $m / z(\%)=330(13)[\mathrm{M}]^{+}, 288(6), 248(18), 247$ (100), $214(20)$, 172 (67), 144 (7), 130 (7), 116 (19), 89 (16).

HRMS (ESI): $m / z$ [M $+\mathrm{H}]^{+}$calcd for $\mathrm{C}_{21} \mathrm{H}_{19} \mathrm{~N}_{2} \mathrm{O}_{2}$ : 331.1441; found: 331.1445 .

\section{1-Acetyl-2-allyl-2-(3-methylbenzyl)indolin-3-one (3m)}

Yield: $26.5 \mathrm{mg}$ (83\%); pale-solid; $\mathrm{mp} 62-64{ }^{\circ} \mathrm{C} ;[\alpha]_{\mathrm{D}}^{24.6}+8.2$ (c 1.10, $\mathrm{CHCl}_{3}$ ); HPLC [Chiralpak AD-H; $i$-PrOH-hexane (5:95); flow rate: 0.7 $\mathrm{mL} / \mathrm{min} ; \lambda=214 \mathrm{~nm}$ ]: $t_{R}=15.5$ (minor), 12.9 (major) $\mathrm{min}$; ee $=68 \%$.

IR (KBr): 3079, 3024, 2961, 2924, 1715, 1670, 1609, 1471, 1436, 1372 , $1300,804,781,702 \mathrm{~cm}^{-1}$.

${ }^{1} \mathrm{H}$ NMR $\left(400 \mathrm{MHz}, \mathrm{CDCl}_{3}\right): \delta=7.63(\mathrm{~d}, J=7.2 \mathrm{~Hz}, 1 \mathrm{H}), 7.43(\mathrm{t}, J=$ $8.0 \mathrm{~Hz}, 1 \mathrm{H}), 7.14(\mathrm{~d}, J=8.0 \mathrm{~Hz}, 1 \mathrm{H}), 7.02(\mathrm{t}, J=7.2 \mathrm{~Hz}, 1 \mathrm{H}), 6.87(\mathrm{t}, J=$ $7.2 \mathrm{~Hz}, 1 \mathrm{H}), 6.78-6.73(\mathrm{~m}, 3 \mathrm{H}), 5.37-5.27(\mathrm{~m}, 1 \mathrm{H}), 5.06(\mathrm{~d}, J=$ $17.2 \mathrm{~Hz}, 1 \mathrm{H}), 4.85(\mathrm{~d}, J=9.6 \mathrm{~Hz}, 1 \mathrm{H}), 3.71(\mathrm{~d}, J=12.8 \mathrm{~Hz}, 1 \mathrm{H}), 3.38$ (dd, $J=12.0,8.0 \mathrm{~Hz}, 1 \mathrm{H}$ ), $3.20(\mathrm{~d}, J=13.2 \mathrm{~Hz}, 1 \mathrm{H}), 3.82$ (dd, $J=11.6$, $8.0 \mathrm{~Hz}, 1 \mathrm{H}), 2.45(\mathrm{~s}, 3 \mathrm{H}), 2.09$ (s, $3 \mathrm{H})$.
${ }^{13} \mathrm{C}$ NMR $\left(150 \mathrm{MHz}\right.$, acetone- $\left.d_{6}\right): \delta=201.9,169.5,153.6,138.0,137.7$, $136.1,132.4,130.9,128.5,128.1,127.3,126.1,124.2,123.9,119.4$, $116.2,77.2,42.0,41.2,27.3,21.2$.

MS (EI): $m / z(\%)=319(35)[\mathrm{M}]^{+}, 278$ (13), 236 (68), 214 (15), 172 (100), 115 (12), 105 (34), 77 (22).

HRMS (ESI): $m / z[M+\mathrm{H}]^{+}$calcd for $\mathrm{C}_{21} \mathrm{H}_{22} \mathrm{NO}_{2}: 320.1645$; found: 320.1637.

\section{1-Acetyl-2-allyl-2-(furan-2-ylmethyl)indolin-3-one (3n)}

Yield: $23.6 \mathrm{mg}$ (80\%); slightly yellow oil; $[\alpha]_{\mathrm{D}}^{24.6}+10.1\left(\right.$ c $\left.1.00, \mathrm{CHCl}_{3}\right)$; HPLC [Chiralpak AD-H; $i$-PrOH-hexane (5:95); flow rate: $0.7 \mathrm{~mL} / \mathrm{min}$; $\lambda=214 \mathrm{~nm}$ ]: $t_{R}=25.0$ (minor), 20.5 (major) $\mathrm{min}$; ee $=76 \%$.

IR (KBr): 3080, 2982, 2853, 1716, 1668, 1607, 1504, 1435, 1371, 1292 , $973,922,750 \mathrm{~cm}^{-1}$.

${ }^{1} \mathrm{H}$ NMR $\left(400 \mathrm{MHz}, \mathrm{CDCl}_{3}\right): \delta=7.70(\mathrm{~d}, J=7.6 \mathrm{~Hz}, 1 \mathrm{H}), 7.52(\mathrm{t}, J=$ $8.0 \mathrm{~Hz}, 1 \mathrm{H}$ ), $7.27(\mathrm{br}, 1 \mathrm{H}), 7.09(\mathrm{t}, J=7.2 \mathrm{~Hz}, 1 \mathrm{H}), 6.95(\mathrm{~s}, 1 \mathrm{H}), 5.98(\mathrm{~s}$, $1 \mathrm{H}), 5.85(\mathrm{~s}, 1 \mathrm{H}), 5.36-5.26(\mathrm{~m}, 1 \mathrm{H}), 5.05(\mathrm{~d}, J=17.2 \mathrm{~Hz}, 1 \mathrm{H}), 4.86$ $(\mathrm{d}, J=10.0 \mathrm{~Hz}, 1 \mathrm{H}), 3.85(\mathrm{~d}, J=9.2 \mathrm{~Hz}, 1 \mathrm{H}), 3.28(\mathrm{br}, 2 \mathrm{H}), 2.77(\mathrm{br}$, $1 \mathrm{H}), 2.48(\mathrm{~s}, 3 \mathrm{H})$.

${ }^{13} \mathrm{C}$ NMR $\left(100 \mathrm{MHz}, \mathrm{CDCl}_{3}\right): \delta=201.4,168.6,150.2,141.6,136.8$, 131.0, 124.9, 124.5, 123.3, 119.6, 114.9, 110.0, 107.9, 75.4, 40.1, 34.3, 27.0.

MS (EI): $m / z(\%)=295(20)\left[\mathrm{M}^{+}, 253(21), 214\right.$ (71), 173 (32), 172 (100), 154 (17), 144 (30), 130 (29), 81 (52).

HRMS (ESI): $m / z[M+\mathrm{H}]^{+}$calcd for $\mathrm{C}_{18} \mathrm{H}_{18} \mathrm{NO}_{3}$ : 296.1281; found: 296.1275 .

\section{Acknowledgment}

This research was financially supported by the Major Basic Research Development Program (Grant No. G 2010CB833300), National Natural Science Foundation of China (21121062, 21032007, 21302205), Chinese Academy of Sciences, Technology Commission of Shanghai Municipality, and the Croucher Foundation of Hong Kong. We thank Dr. Bai-Lin Lei for inspiring discussions.

\section{Supporting Information}

Supporting information for this article is available online at http://dx.doi.org/10.1055/s-0034-1379043.

\section{References}

(1) (a) Steyn, P. S. Tetrahedron Lett. 1971, 3331. (b) Steyn, P. S. Tetrahedron 1973, 29, 107. (c) Hutchison, A. J.; Kishi, Y. J. Am. Chem. Soc. 1979, 101, 6786.

(2) (a) Birch, A. J.; Wright, J. J. J. Chem. Soc., Chem. Commun. 1969, 644. (b) Birch, A. J.; Wright, J. J. Tetrahedron 1970, 26, 2329. (c) Birch, A. J.; Russell, R. A. Tetrahedron 1972, 28, 2999. (d) Williams, R. M.; Glinka, T.; Kwast, E.; Coffman, H.; Stille, J. K. J. Am. Chem. Soc. 1990, 112, 808.

(3) (a) Bhakuni, D. S.; Silva, M.; Matlin, S. A.; Sammes, P. G. Phytochemistry 1976, 15, 574. (b) Stoermer, D.; Heathcock, C. H.J. Org. Chem. 1993, 58, 564.

(4) (a) Liu, J.-F.; Jiang, Z.-Y.; Wang, R.-R.; Zeng, Y.-T.; Chen, J.-J.; Zhang, X.-M.; Ma, Y.-B. Org. Lett. 2007, 9, 4127. (b) Karadeolian, A.; Kerr, M. A. Angew. Chem. Int. Ed. 2010, 49, 1133. (c) Karadeolian, A.; Kerr, M. A. J. Org. Chem. 2010, 75, 6830. 
(d) Lee, J.; Panek, J. S. Org. Lett. 2011, 13, 502. (e) Zhang, X.; Mu, T.; Zhan, F. X.; Ma, L. J.; Liang, G. X. Angew. Chem. Int. Ed. 2011, 50,6164 .

(5) (a) Blackman, A. J.; Hambly, T. W.; Picker, K.; Taylor, W. C.; Thirasasana, N. Tetrahedron Lett. 1987, 28, 5561. (b) Liu, Y.; McWhorter, W. W. J. Am. Chem. Soc. 2003, 125, 4240. (c) Higuchi, K.; Sato, Y.; Tsuchimochi, M.; Sugiura, K.; Hatori, M.; Kawasaki, T. Org. Lett. 2009, 11, 197.

(6) (a) Takahashi, I.; Takahashi, K.; Ichimura, M.; Morimoto, M.; Asano, K.; Kawamoto, I.; Tomita, F.; Nakano, H. J. Antibiot. 1988, 41, 1915. (b) Fukuda, Y.; Nakatani, K.; Ito, Y.; Terashima, S. Tetrahedron Lett. 1990, 31, 6699.

(7) For racemic versions, see selected examples: (a) Lednicer, D.; Emmert, D. E. J. Heterocycl. Chem. 1970, 7, 575. (b) Zhang, X.; Foote, C. S. J. Am. Chem. Soc. 1993, 115, 8867. (c) Tommasi, G.; Bruni, P.; Greci, L.; Sgarabotto, P.; Righi, L. J. Chem. Soc., Perkin Trans. 1 1999, 681. (d) Liu, Y.; McWhorter, W. W. Jr.J. Org. Chem. 2003, 68, 2618. (e) Boger, D. L.; Nishi, T. Bioorg. J. Med. Chem. 1995, 3, 67. (f) Yun, S.; Kim, K. J. Chem. Soc., Perkin Trans. 12002 , 2360. (g) Buller, M. J.; Cook, T. G.; Kobayashi, Y. Heterocycles 2007, 72, 163. (h) Gowan, M.; Caille, A. S.; Lau, C. K. Synlett 1997, 1312. (i) Merour, J.-Y.; Chichereau, L.; Desarbre, E.; Gadonneix, P. Synthesis 1996, 519. (j) Kamel, A. A.; Abdou, W. M. Synlett 2007, 1269. (k) Yamada, K.; Kur-okawa, T.; Tokuyama, H.; Fukuyama, T. J. Am. Chem. Soc. 2003, 125, 6630. (1) Astolfi, P.; Bruni, P.; Greci, L.; Stipa, P.; Righi, L.; Rizzoli, C. Eur. J. Org. Chem. 2003, 2626. For asymmetric and catalytic versions, see: $(\mathrm{m}) \mathrm{Li}$, L.; Han, M.; Xiao, M.; Xie, Z. Synlett 2011, 1727. (n) Rueping, M.; Raja, S.; Nuñez, A. Adv. Synth. Catal. 2011, 353, 563. (o) Parra, A.; Alfaro, R.; Marzo, L.; Moreno-Carrasco, A.; Garcia Ruano, J. L.; Aleman, J. Chem. Commun. 2012, 48, 9759. (p) Rueping, M.; Rasappan, R.; Raja, S. Helv. Chim. Acta 2012, 95, 2296. (q) Liu, J.X.; Zhou, Q.-Q.; Deng, J.-G.; Chen, Y.-C. Org. Biomol. Chem. 2013, 11, 8175. (r) Rueping, M.; Raja, S. Beilstein J. Org. Chem. 2012, 8, 1819. (s) Yin, Q.; You, S.-L. Chem. Sci. 2011, 3, 1344. (t) Higuchi, K.; Masuda, K.; Koseki, T.; Hatori, M.; Sakamoto, M.; Kawasaki, T. Heterocycles 2007, 73, 641.
(8) For reviews, see: (a) Trost, B. M.; Van Vranken, D. L. Chem. Rev. 1996, 96, 395. (b) Pfaltz, A.; Lautens, M. In Comprehensive Asymmetric Catalysis; Vol. 2; Jacobsen, E. N.; Pfaltz, A.; Yamamoto, H., Eds.; Springer: New York, 1999, 833. (c) Trost, B. M.; Crawley, M. L. Chem. Rev. 2003, 103, 2921. (d) Lu, Z.; Ma, S. Angew. Chem. Int. Ed. 2008, 47, 258. (e) Ding, C.-H.; Hou, X.-L. Top. Organomet. Chem. 2011, 36, 247.

(9) (a) Genet, J. P.; Ferroud, D.; Juge, S.; Monte, J. R. Tetrahedron Lett. 1986, 27, 4573. (b) Nakoji, M.; Kanayama, T.; Okino, T.; Takemoto, Y. Org. Lett. 2001, 3, 3329. (c) Kuwano, R.; Ito, Y. J. Am. Chem. Soc. 1999, 121, 3236. (d) Kuwano, R.; Nishio, R.; Ito, Y. Org. Lett. 1999, 1, 837. (e) Wang, Y. Ph.D. Dissertation; Shanghai Institute of Organic Chemistry: P. R. of China, 2002. (f) Maity, P.; König, B. Synthesis 2006, 2719. (g) Nemoto, T.; Harada, T.; Matsumoto, T.; Hamada, Y. Tetrahedron Lett. 2007, 48, 6304. (h) Malinowski, J. T.; McCarver, S. J.; Johnson, J. S. Org. Lett. 2012, 14, 2878. (i) Ogasawara, M.; Ngo, H. L.; Sakamoto, T.; Takahashi, T.; Lin, W. Org. Lett. 2005, 7, 2881.

(10) (a) You, S.-L.; Zhu, X.-Z.; Luo, Y.-M.; Hou, X.-L.; Dai, L.-X. J. Am. Chem. Soc. 2001, 123, 7471. (b) Sun, N.; Hou, X.-L. Org. Lett. 2004, 6, 4399. (c) Zheng, W.-H.; Sun, N.; Hou, X.-L. Org. Lett. 2005, 7, 5151. (d) Zheng, W.-H.; Zheng, B.-H.; Zhang, Y.; Hou, X.L. J. Am. Chem. Soc. 2007, 129, 7718. (e) Zhang, K.; Peng, Q.; Hou, X.-L.; Wu, Y.-D. Angew. Chem. Int. Ed. 2008, 47, 1741. (f) Chen, J.P.; Ding, C.-H.; Liu, W.; Hou, X.-L.; Dai, L.-X. J. Am. Chem. Soc. 2010, 132, 15493. (g) Zheng, B.-H.; Ding, C.-H.; Hou, X.-L. Synlett 2011, 2262. (h) Yang, X.-F.; Ding, C.-H.; Li, X.-H.; Huang, J.-Q.; Hou, X.-L.; Dai, L.-X.; Wang, P.-J. J. Org. Chem. 2012, 77, 8980. (i) Li, X.-H.; Zheng, B.-H.; Ding, C.-H.; Hou, X.-L. Org. Lett. 2013 , 15, 6086.

(11) Reviews: (a) Fuji, K. Chem. Rev. 1993, 93, 2037. (b) Corey, E. J.; Guzman-Perez, A. Angew. Chem. Int. Ed. 1998, 37, 388. (c) O'Brien, P. J. Chem. Soc., Perkin Trans. 1 2001, 95. (d) Christoffers, J.; Mann, A. Angew. Chem. Int. Ed. 2001, 40, 4591. (e) Christoffers, J.; Baro, A. Adv. Synth. Catal. 2005, 347, 1473. (f) Trost, B. M.; Jiang, C. Synthesis 2006, 369. (g) Bella, M.; Casperi, T. Synthesis 2009, 1583. (h) Hong, A. Y.; Stoltz, B. M. Eur. J. Org. Chem. 2013, 2745.

This article differs from the e-first online version only in its layout; no content has been changed. 